\title{
Preclinical models in radiation oncology
}

\author{
Jenna Kahn ${ }^{1,2}$, Philip J Tofilon ${ }^{1}$ and Kevin Camphausen ${ }^{1 *}$
}

\begin{abstract}
As the incidence of cancer continues to rise, the use of radiotherapy has emerged as a leading treatment modality. Preclinical models in radiation oncology are essential tools for cancer research and therapeutics. Various model systems have been used to test radiation therapy, including in vitro cell culture assays as well as in vivo ectopic and orthotopic xenograft models. This review aims to describe such models, their advantages and disadvantages, particularly as they have been employed in the discovery of molecular targets for tumor radiosensitization. Ultimately, any model system must be judged by its utility in developing more effective cancer therapies, which is in turn dependent on its ability to simulate the biology of tumors as they exist in situ. Although every model has its limitations, each has played a significant role in preclinical testing. Continued advances in preclinical models will allow for the identification and application of targets for radiation in the clinic.
\end{abstract}

Keywords: Preclinical models, Radiation oncology, Radiosensitizer, Orthotopic xenograft model

\section{Background}

It is estimated that in 2012 that there will be 1.6 million non-skin cancers diagnosed in North America; nearly three fourths of these cancer patients will receive radiation therapy sometime during the course of their illness [1]. Because radiotherapy continues to serve as a primary cancer treatment modality, the development of strategies that improve its efficacy could benefit a significant number of patients. Whereas most improvements in tumor control achieved in the past 30 years of radiation oncology can be attributed to advances in dose delivery technology, further improvements will likely depend on a greater understanding of the biology underlying tumor response to radiation. More specifically, it is generally considered that the delineation of the fundamental mechanisms mediating tumor cell radioresistance will lead to the identification of molecules that can serve as targets for radiosensitization. Such information will provide the basis for the development of clinical protocols combining molecularly targeted agents and radiotherapy.

Towards this end, laboratory studies have implicated more than 70 molecules as potential determinants of tumor cell radiosensitivity, with additional molecules identified every year [2]. Thus, there is no shortage of putative

\footnotetext{
* Correspondence: camphauk@mail.nih.gov

'Radiation Oncology Branch, National Cancer Institute, National Institutes of Health, 10 Center Drive, Building 10, CRC Rm B2-3561, Bethesda, MD 20892, USA

Full list of author information is available at the end of the article
}

molecular targets for radiosensitization. However, translation of these data into clinically relevant targets for cancer radiotherapy requires more than establishing a causal relationship between a molecule and the radiosensitivity of a given tumor cell line: additional information is required. Clearly, whether the target is selective for the radiosensitization of tumor over normal cells should be determined. An additional and more difficult question, regards cellular context. It is often the case for tumor selective targets that they influence the radiosensitivity of some tumor cells, but not others. Defining the genetic, epigenetic and microenvironmental circumstances, i.e. cellular context, in which a molecule functions as a determinant of radiosensitivity would be of considerable value in the development of successful clinical applications. Establishing causal relationships, tumor selectivity and cellular context in a relevant preclinical setting requires the use of a number of experimental models. The goal of this review is to describe the model systems currently employed in research efforts aimed at developing molecular targets for tumor radiosensitization.

\section{In Vitro culture systems}

Cells grown in monolayer cultures provide an experimentally expedient model for cancer research in general and have been used extensively in defining the molecular determinants of radiosensitivity. Cell culture experiments typically comprise the initial step in establishing a
C Biomed Central

(c) 2012 Kahn et al.; licensee BioMed Central Ltd. This is an Open Access article distributed under the terms of the Creative Commons Attribution License (http://creativecommons.org/licenses/by/2.0), which permits unrestricted use, distribution, and reproduction in any medium, provided the original work is properly cited. 
causal relationship between a suspected molecular determinant and radiosensitivity as well as the preliminary evaluation of drug/radiation combinations. In this model system radiosensitivity is most rigorously defined using the clonogenic survival assay, which measures the proliferative (clonogenic) capacity of individual cells. In the most commonly used form of this assay, single cell suspensions are seeded into tissue culture plates, allowed to attach and irradiated; colonies are then counted after 10-21 days, depending on the specific cell line [3]. The minimum colony size is typically set at 50 cells, which coincides with 5-6 doublings. The necessity for colony formation, which involves a relatively prolonged time period between irradiation and analysis of cell death (defined as the loss of reproductive potential), is based on the fundamental mechanisms of radiobiology. For most solid tumor cells, as well as for fibroblasts, the primary mode of cell death after radiation exposure is mitotic catastrophe, which can require several cell divisions for maximal expression. Moreover, radiation induces transient growth arrest, which can be misinterpreted as cytotoxicity in short term assays based on cell number. With respect to detecting modifications in radiosensitivity, targeting of molecular determinants can potentially switch the mode of death from mitotic catastrophe to apoptosis or autophagy; each form of death is accounted for in the clonogenic survival assay. Thus, the gold standard for quantifying radiosensitivity and its modification is clonogenic survival analysis.

An additional advantage of cell culture models is the capacity for the molecular manipulation of suspected determinants of radiosensitivity (Figure 1). Standard molecular techniques using genetic and epigenetic approaches have traditionally been combined with the clonogenic survival assay to establish causal relationships between a suspected target and radiosensitization. Furthermore, analyses of such processes as cell cycle phase distribution, DNA DSB induction and repair, and of the mode cell death (apoptosis, mitotic catastrophe or autophagy) provide insight into the mechanism through which the molecule in question affects radioresponse. Cell culture models have also been used in the initial evaluation of whether a molecularly targeted agent acts in a predictable fashion. For example, our lab has previously performed experiments using the HDAC inhibitor valproic acid [4]. Using immunoblots generated from monolayer cultures, valproic acid was shown to induce hyper-acetylation of histone $\mathrm{H} 3$ or $\mathrm{H} 4$, and this hyperacetylation was dependant on continuous drug exposure. Clonogenic survival analysis then showed that continuous exposure of cells to valproic acid was necessary to achieve the maximum enhancement of radiation induced cell death, consistent with the dependence on continuous drug exposure exhibited by histone hyperacetylation.

Numerous studies aimed at defining the molecular determinants of radiosensitivity have initially been performed using in vitro monolayer cultures. Comparison of tumor cell lines to normal cell lines such as fibroblasts or mammary epithelial cells provides for an initial evaluation of whether a molecular determinant and/or a targeted agent selectively enhance the radiosensitivity of tumor cells over normal cells. Using multiple tumor cell lines across various histologies may provide a system for investigating cell the genetic and epigenetic context under which a molecule regulates radiosensitivity.

Although essential to establishing causal relationships between a molecule and radiosensitivity, i.e., identifying molecular targets, the use of in vitro model systems is not without limitations. First, the use of in vitro systems is based on the assumption that the phenotype of tumor cells in culture recapitulates that of cells in an in vivo

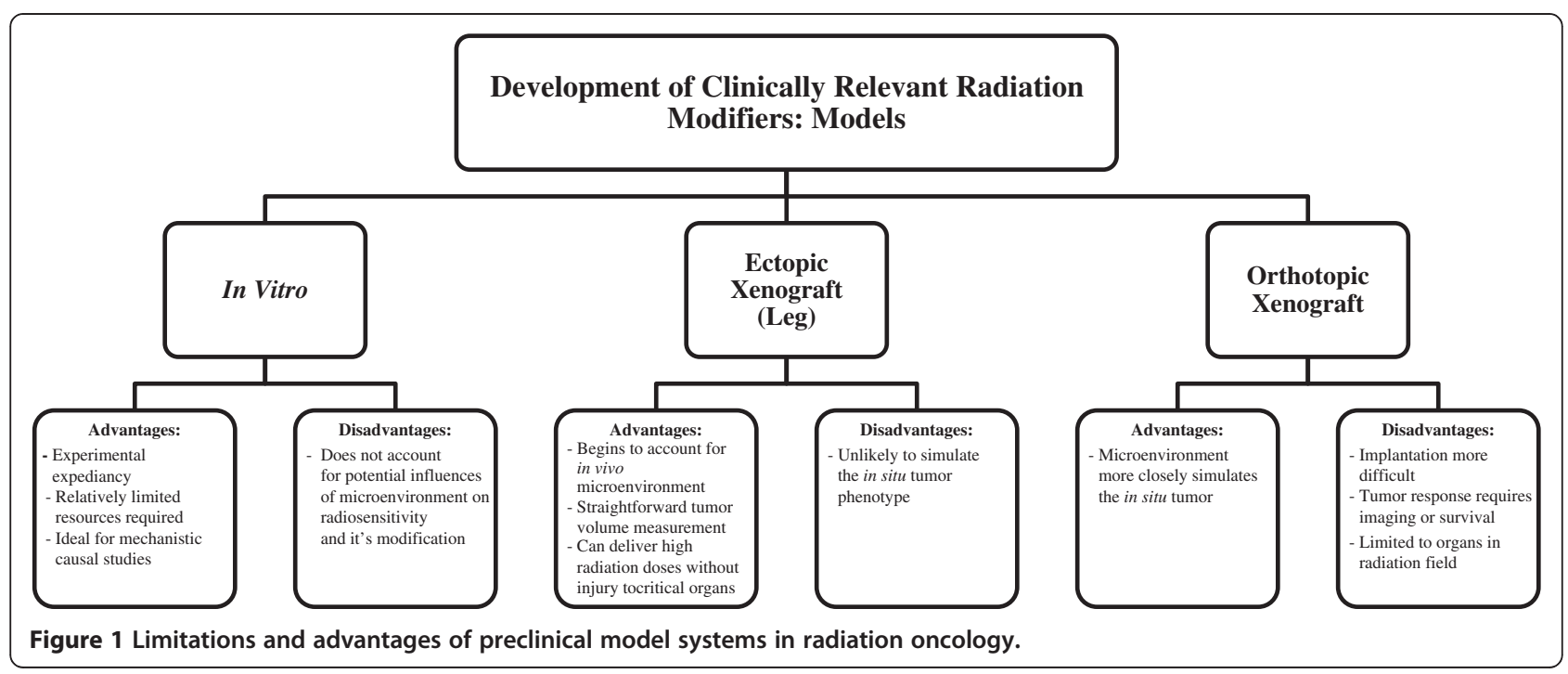


setting, i.e. the same targets are expressed and are operative. Many commonly used human tumor cell lines have been maintained on plastic in the laboratory for years, allowing for additional unwanted selection pressures; thus, the cell lines may not be representative of the original tumor in situ. In a recent study, Shai et al. showed by microarray analysis that tumor cells grown for three passages on plastic were no longer representative of the in situ tumors from which they were derived [5]. Furthermore, cells grown in vitro lack the architectural and cellular complexity of in vivo tumors including inflammatory, vascular, and stromal components [6], factors that can influence tumor radioresponse.

\section{In vivo models}

Both rodent and human cells have been used to investigate in vivo tumor radioresponse. Most rodent cell studies have focused on implanted murine or rat syngeneic tumors, although more recently genetically engineered mouse (GEM) models have been used to evaluate the radioresponse of spontaneous tumors [7-9]. The primary model for investigating the radioresponse of human tumor cells under in vivo conditions involves their implantation and growth in immunocompromised mice or rats [10]. Whereas both rodent and human tumor models are available, studies aimed at developing molecularly targeted radiosensitizing agents have been primarily performed using human tumor cells. The rationale, as recently summarized by Olive and colleagues, is that there are significant differences between rodent and human cells in terms of the fundamental processes regulating radioresponse [11]. Studies performed over the years have shown that the activities and/or levels of critical molecules mediating the DNA damage response after irradiation such as DNA-PK, Ku70, Ku86 and NBS differ between species. Moreover, in terms of general processes, rodent cells are more susceptible to radiationinduced oncogenic transformation, less efficient at checkpoint activation, and more sensitive to oxidative stress as compared to human cells. Given the differences between rodent and human cells regarding aspects of the fundamental mechanisms mediating cellular response and given that a goal of experimental radiation oncology is to develop modifiers relevant to treatment, most preclinical radiation studies focus on human tumor xenografts. However, it should be noted that the human tumor xenograft model does have a number of potentially significant limitations, which include the absence of an immune response and the presence of mouse rather than human stroma. Clearly, depending on the molecular target being addressed and its mechanism of action, these characteristics need to be taken into account when attempting to translate results to a clinical situation.
The most common assay used in defining in vivo radioresponse is the tumor growth delay (TGD) assay [3]. Typically, tumor cells, regardless of tissue of origin, are implanted subcutaneously on the leg. This site, in contrast to the flank, allows for delivery of radiation to the tumor without exposing critical organs. After implantation, the tumor is allowed to grow to a measurable volume, the mice are randomized, and therapy is initiated. Tumor volumes are then measured 2-3x weekly with data plotted as the mean of the volumes. However, each tumor should be measured and tracked separately, as the TGD is calculated for each tumor and reported as the difference between the control and experimental group as days delay $+/$ - SEM. Advantages of the leg TGD model include expedient implantation, relatively straightforward volume measurements and minimal impact on the overall wellbeing of the mouse, which limits the introduction of complicating variables. The primary disadvantage is that the microenvironment does not simulate that of the in situ tumor.

To account for the potential influence of tumor specific microenvironments, another in vivo model used in experimental radiation oncology is the orthotopic implant of tumor cells. This technique has been applied to the radiation response of pancreas, lung, breast, prostate and brain tumors. Our laboratory focuses on brain tumors, as such; we commonly do orthotopic implants of human GBM cells into the brains of nude mice [12]. To perform this technique, a stereotactic apparatus is used to implant tumor cells into a specific area of the brain. Typically, bioluminescent imaging or magnetic resonance imaging can be used to confirm both presence and volume of tumor before initiating the experimental therapy. Irradiation is performed using a customized jig that allows irradiation of the tumor but shields the remainder of the mouse. Once the therapy has begun, the most common endpoint is survival and a Kaplan-Meier curve is generated to determine significance of each therapy. The main advantage of the use of orthotopic tumors is that the microenvironment may more closely represent that of the in situ tumor. The disadvantages are the difficultly and skill required to perform the implants, the difficulty of imaging tumors and the impact the tumor implant has on the organ function of the mouse.

\section{In vitro vs. ectopic vs. orthotopic models}

As described above, each model system used in the development of molecular targets for radiosensitization has advantages and disadvantages. However, the ultimate utility of a given model to the development of more effective cancer therapies depends on its ability to simulate the phenotype of tumor cells as they exist in situ. To begin to address this issue, our lab has used gene expression profiling as an indicator of phenotype to 
compare two human glioma cell lines (U87 and U251) grown in vitro as a monolayer and in vivo as subcutaneous leg and as intracranial xenograft tumors [13]. For each cell line, the gene expression profile generated from tissue culture was significantly different from that generated from the subcutaneous tumor, which was significantly different from those grown intracranially (i.c.). The U251 and U87 gene expression profiles generated under the three growth conditions were also compared to one another. As expected, the profiles of the two glioma cell lines were significantly different from each other when grown as monolayer cultures. However, the expression profiles for the glioma cell lines were less discordant when grown as subcutaneous tumors, and actually similar when grown as intracranial tumors. Using Statistical Analysis of Microarray software, we also showed that the two cell lines had 290 genes that were common outliers in the orthotopic environment compared to the in vitro growth environment (SAM ref). Using the GOStat program, the 290 outlier genes were mapped to functions of central nervous system development and CNS function (GoSTAT ref). These data suggest that the microenvironment has a significant impact on the phenotype of the tumor model and thus the potential response to cytotoxic therapy. This work has been expanded, by additional investigators, to tumors from different in situ environments including model systems of ovarian tumors, meduloblastoma, head and neck cancers and lung cancers [14-17].

To determine if the tumor model microenvironment had an effect on the response to radiation therapy we next irradiated U87 and U251 cells as monolayer cultures, as ectopic flank tumors and as orthotopic tumors and collected the tumors for cDNA microarray analysis [18]. The comparison of the arrays for the in vitro samples showed very few changes at the mRNA level as well as very few common changes between the cell lines. However, in the samples from the orthotopic tumors there were over 700 common genes that significantly changed after therapy in both cell lines. These data suggest that whereas genotype may be the overwhelming determinant of radiosensitivity for cultured cells, under i.c. conditions the brain microenvironment plays a significant role in regulating the genes affected by radiation. Similar results have been obtained by other investigators using an alternate GBM model as well as an orthotopic pancreas model $[19,20]$. Thus, taking into account such environmental influences will likely be critical in defining the putative functional significance of radiation-induced changes in gene expression when comparing different model systems.

However, results showing the differences between model systems do not directly address the central question of which model system is most similar to the in situ tumors. Currently, there is no biopsy data from patients undergoing radiotherapy after one or several doses of radiation. However, several datasets exist of resected GBM samples studied by microarray profiling [21,22] Shankavaram and colleagues recently completed a comparison of three model systems and the clinical dataset published by Bredel et al. [23]. The data from both the in vitro and ectopic in vivo samples were not highly correlated with any of the primary GBM samples. However, the data from the orthotopic samples was highly correlated to a subset of the entire clinical database that involved genes and pathways related to neurogenesis and growth factor-induced signal transduction. Moreover, using the combined clinical and experimental datasets, glutamate receptor was suggested as a potential target for radiosensitization unique to the orthotopic system. That is, treatment with the glutamate receptor inhibitor LY341595 was shown to enhance the radiosensitivity of glioma cells grown in the orthotopic model, but not in either the in vitro or ectopic tumor models. Thus, these results suggest that the orthotopic tumors represented a subset of the samples collected from patients with GBM and that this subset can be used to discover a novel target for GBM radiosensitization, information that could not be generated from the more standard glioma models of in vitro monolayer culture and subcutaneous xenografts.

\section{Conclusion}

The current state of the art in preclinical modeling in radiation oncology research involves the use of in vitro cultures and assays, as well as ectopic and orthotopic xenograft models. As investigators initiate pre-clinical experiments, analysis of the genetics of the cell lines used the composition of the microenvironment and the experimental techniques available should be evaluated and compared to the proposed disease to be treated in the clinic.

\section{Competing interests}

The authors declare that they have no competing interests.

\section{Authors' contributions}

All of the authors participated in the writing, reviewing, and editing of the manuscript. All authors read and approved the final manuscript.

\section{Author details}

1 Radiation Oncology Branch, National Cancer Institute, National Institutes of Health, 10 Center Drive, Building 10, CRC Rm B2-3561, Bethesda, MD 20892, USA. ${ }^{2}$ Warren Alpert School of Medicine at Brown University, Providence, RI, USA

Received: 11 December 2012 Accepted: 18 December 2012 Published: 27 December 2012

\section{References}

1. SEER: Surveillance epidemiology and End results (SEER), national cancer institiute. www.cancergov.

2. Tofilon PJ, Camphausen K: Molecular targets for tumor radiosensitization Chem Rev 2009, 109(7):2974-2988.

3. Hall EJ: Radiobiology for the radiologist. 7th edition. Philadelphia: Lippincott Williams and Wilkins; 2011. 
4. Camphausen K, Cerna D, Scott T, Sproull M, Burgan WE, Cerra MA, Fine H, Tofilon PJ: Enhancement of in vitro and in vivo tumor cell radiosensitivity by valproic acid. Int J Cancer J Int du cancer 2005, 114(3):380-386.

5. Mehrian Shai R, Reichardt JK, Ya-Hsuan H, Kremen TJ, Liau LM, Cloughesy TF, Mischel PS, Nelson S, Mehrian Shai R, Reichardt JK, Ya-Hsuan H, Kremen TJ, Liau LM, Cloughesy TF, Mischel PS, Nelson SF: Robustness of gene expression profiling in glioma specimen samplings and derived cell lines. Brain Res Mol Brain Res 2005, 136(1-2):99-103.

6. McAllister SS, Weinberg RA: Tumor-host interactions: a far-reaching relationship. J Clin Oncol 2010, 28(26):4022-4028.

7. Fomchenko El, Holland EC: Mouse models of brain tumors and their applications in preclinical trials. Clin Cancer Res 2006, 12(18):5288-5297.

8. Kirsch DG, Santiago PM, di Tomaso E, Sullivan JM, Hou WS, Dayton T, Jeffords LB, Sodha P, Mercer KL, Cohen R, et al: p53 Controls radiationinduced gastrointestinal syndrome in mice independent of apoptosis. Science 2010, 327(5965):593-596.

9. Yoon SS, Stangenberg L, Lee YJ, Rothrock C, Dreyfuss JM, Baek KH Waterman PR, Nielsen GP, Weissleder R, Mahmood U, et al: Efficacy of sunitinib and radiotherapy in genetically engineered mouse model of soft-tissue sarcoma. Int J Radiat Oncol Biol Phys 2009, 74(4):1207-1216.

10. Rygaard J, Povlsen CO: Heterotransplantation of a human malignant tumour to "nude" mice. Acta Pathol Microbiol Scand 1969, 77(4):758-760.

11. Banuelos CA, Banath JP, MacPhail SH, Zhao J, Eaves CA, O'Connor MD, Lansdorp PM, Olive PL: Mouse but not human embryonic stem cells are deficient in rejoining of ionizing radiation-induced DNA double-strand breaks. DNA Repair 2008, 7(9):1471-1483.

12. Kramp TR, Camphausen K: Combination radiotherapy in an orthotopic mouse brain tumor model. JOVE 2012, 61:e3397.

13. Camphausen K, Purow B, Sproull M, Scott T, Ozawa T, Deen DF, Tofilon PJ: Influence of in vivo growth on human glioma cell line gene expression: convergent profiles under orthotopic conditions. Proc Natl Acad Sci U S A 2005, 102(23):8287-8292.

14. Greenaway J, Moorehead R, Shaw P, Petrik J: Epithelial-stromal interaction increases cell proliferation, survival and tumorigenicity in a mouse model of human epithelial ovarian cancer. Gynecol Oncol 2008, 108 (2):385-394.

15. Annabi B, Rojas-Sutterlin S, Laflamme C, Lachambre MP, Rolland Y, Sartelet H, Beliveau R: Tumor environment dictates medulloblastoma cancer stem cell expression and invasive phenotype. Mol Cancer Res: MCR 2008, 6(6):907-916.

16. Gelbard A, Kupferman ME, Jasser SA, Chen W, El-Naggar AK, Myers JN, Hanna EY: An orthotopic murine model of sinonasal malignancy. Clin Cancer Res 2008, 14(22):7348-7357.

17. Li B, Torossian A, Li W, Schleicher S, Niu K, Giacalone NJ, Kim SJ, Chen H, Gonzalez A, Moretti L, et al: A novel bioluminescence orthotopic mouse model for advanced lung cancer. Radiat Res 2011, 176(4):486-493.

18. Camphausen K, Purow B, Sproull M, Scott T, Ozawa T, Deen DF, Tofilon PJ: Orthotopic growth of human glioma cells quantitatively and qualitatively influences radiation-induced changes in gene expression. Cancer Res 2005, 65(22):10389-10393.

19. Lamfers ML, Idema S, Bosscher L, Heukelom S, Moeniralm S, van der

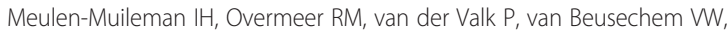
Gerritsen WR, et al: Differential effects of combined Ad5- delta 24RGD and radiation therapy in in vitro versus in vivo models of malignant glioma. Clin Cancer Res 2007, 13(24):7451-7458.

20. Lee CJ, Spalding AC, Ben-Josef E, Wang L, Simeone DM: In vivo bioluminescent imaging of irradiated orthotopic pancreatic cancer xenografts in nonobese diabetic-severe combined immunodeficient mice: a novel method for targeting and assaying efficacy of ionizing radiation. Transl Oncol 2010, 3(3):153-159.

21. Bredel M, Bredel C, Juric D, Harsh GR, Vogel H, Recht LD, Sikic Bl: Highresolution genome-wide mapping of genetic alterations in human glial brain tumors. Cancer Res 2005, 65(10):4088-4096.

22. Comprehensive genomic characterization defines human glioblastoma genes and core pathways. Nature 2008, 455(7216):1061-1068.

23. Shankavaram UT, Bredel M, Burgan WE, Carter D, Tofilon P, Camphausen K: Molecular profiling indicates orthotopic xenograft of glioma cell lines simulate a subclass of human glioblastoma. J Cell Mol Med 2012, 16(3):545-554.

\section{Submit your next manuscript to BioMed Central and take full advantage of:}

- Convenient online submission

- Thorough peer review

- No space constraints or color figure charges

- Immediate publication on acceptance

- Inclusion in PubMed, CAS, Scopus and Google Scholar

- Research which is freely available for redistribution 